\section{Kniegelenkarthrose: Der Depressions-Phänotyp beeinflusst den Krankheitsverlauf}

Rathbun AM et al. Depression Subtypes in Individuals With or at Risk for Symptomatic Knee Osteoarthritis. Arthritis Care Res (Hoboken) 2020; 72: 669-678. doi:10.1002/acr.23898

Viele Patienten mit einer Kniegelenkarthrose entwickeln im Krankheitsverlauf depressive Symptome, welche sich wiederum ungünstig auf die Gelenkproblematik auswirken können. Welchen verschiedenen Depressions-Subtypen Arthrosepatienten bzw. -Risikopersonen angehören und inwiefern sich diese Subtypen auf Schmerzbelastungen und Einschränkungen auswirken, untersuchten nun USWissenschaftler.

Sie werten die Daten von 4486 Personen aus, die an der Osteoarthritis Initiative (OAI), einer Beobachtungsstudie zur Identifikation von Risikofaktoren und Biomarkern bezüglich des Neuauftretens oder Voranschreitens der Gonarthrose, teilgenommen hatten. 1626 Individuen galten bei Studieneinschluss als Arthrose-Risikopersonen und 2860 litten an einer symptomatischen Arthose. Alle Studienteilnehmer beantworteten initial die 20 Items umfassende Center for Epidemiologic Studies Depression Scale (CES-D). Anhand des hiermit erfassten Spektrums depressiver Symptome identifizierten die Forscher Personen mit ähnlichem Symptommuster. Anschließend prüften sie, welcher Zusammenhang zwischen diesen verschiedenen Depressions-Phänotypen und den nach 4 Jahren mittels WOMAC (Western Ontario and McMaster Universities Arthritis Index) objektivierten Endpunkten „Schmerzen“ und „Behinderungen“ bestand.

\section{Ergebnisse}

Im Studienkollektiv identifizierten die Forscher 4 Depressions-Subtypen: Den asymptomatischen Typ (80,6\%), dem katatonen Typ (5,3\%), den anhedonistischen Typ $(10,6 \%)$ sowie den melancholischen Typ (3,5\%). Personen vom asymptomatischen Typ hatten die niedrigsten, Personen vom melancholischen Typ dagegen die höchsten
CES-D-Scores. Die Personen der beiden anderen Typen wiesen ähnlich hohe, mittlere Punktwerte auf. Die Individuen mit einer anhedonistischen Symptomdominanz zeichneten sich durch die Unfähigkeit, Freude zu empfinden, aus. Beim katatonen Subtyp standen eine psychomotorische Agitation sowie somatische Beschwerden (Energieund Bewegungsarmut, Konzentrationsschwierigkeiten, ruheloser Schlaf) im Vordergrund. Das breiteste Symptomspektrum beobachteten die Forscher beim melancholischen Subtyp (Traurigkeit, Einsamkeit, Anhedonie, psychomotorische Agitation, somatische Symptome). Bezüglich des Arthroseverlaufs zeigte sich: Im Vergleich zum asymptomatischen Depressions-Typ wiesen die Personen vom anhedonistischen und melancholische Subtyp stärkere Schmerzbelastungen und Einschränkungen auf, wobei die Patienten mit manifester Kniegelenkarthrose diesbezüglich deutlicher betroffen waren als die Risikopersonen.

\section{FAZIT}

Gonarthrosepatienten sowie Personen mit diesbezüglicher Risikokonstellation, so das Fazit der Autoren, lassen sich 4 differenzierten Depressions-Phänotypen zuordnen, welche sich durch bestimmte Symptommuster auszeichnen und den Arthroseverlauf in unterschiedlichem Ausmaß beeinflussen. Sie halten daher ein entsprechendes Depressionsscreening für Kniearthrosepatienten für sinnvoll. Auf der Basis dieser Diagnostik könnten anschließend personalisierte psychische Interventionen eingeleitet werden.

\section{Dr. med. Judith Lorenz, Künzell}

